\title{
Kaposi Sarcoma in a Hiv Uninfected Man who Has Sex with Men
}

\author{
A. Potthoff ${ }^{1}$, N. H. Brockmeyer ${ }^{1}$, M. Stücker ${ }^{1}$, U. Wieland ${ }^{2}$, A. Kreuter ${ }^{1}$ \\ for the Competence Network HIV/AIDS \\ ${ }^{1}$ Department of Dermatology, Ruhr-University Bochum, Germany \\ ${ }^{2}$ Institute of Virology, National Reference Center for Papilloma- and Polyomaviruses, Cologne, Germany
}

\begin{abstract}
Kaposi's sarcoma (KS) is a rare angioproliferative tumor associated with human herpesvirus 8 (HHV-8) infection. Four clinical variants of KS have been described: classic, endemic, iatrogenic and HIV-associated. We describe a 53-year-old men who had sex with men with a rapidly growing nodule on his left foot. Histologically KS was confirmed. Our patient did not match the clinical subgroups as HIV infection or other immune disorders could be ruled out. KS in HIV-negative MSM has only been reported sporadically. It was shown that KS in these patients clinically resembles classic KS but occurs at a younger age, is limited to the skin, and is associated with a good prognosis.
\end{abstract}

Key words: Kaposi sarcoma, human herpesvirus 8, men having sex with men, HIV-infection

\section{INTRODUCTION}

Kaposi's sarcoma (KS) is a rare angioproliferative tumor associated with human herpesvirus 8 (HHV-8) infection. Four clinical variants of KS exist: classic (predominantly located at the lower extremities of older men from Mediterranean areas), endemic (in younger Africans with frequent local invasive and/or visceral disease), iatrogenic (in organ recipients on immunosupression), and HIV-associated (often presenting with multifocal skin lesions and frequent visceral disease). The latter mainly affects HIV-positive men having sex with men (MSM). We herein report a HIV-negative MSM with KS who does not match with these 4 clinical subgroups.

\section{CASE REPORT}

A 53-year old German patient presented with a rapidly growing nodule of about $1 \mathrm{~cm}$ in diameter located on his left foot (Fig. 1). Histo-pathological evaluation of the lesion revealed a strong proliferation of spindleshaped cells outlining irregular slits and clefts, consistent with $\mathrm{KS}$. Immunohistological staining for $\mathrm{HHV}-8$ showed strong immunopositivity (Fig. 2). The patient reported three similar lesions on his chest 6 years previously that also turned out to be KS. Several HIV tests performed within the last years were negative, and re-testing at our institution was negative as well. There were no abnormalities in the differential blood and $\mathrm{B}$ and $\mathrm{T}$ cell subcounts. $\mathrm{CD}^{+}$cells were $563 / \mu \mathrm{l}$ (range: $410-1590 / \mu \mathrm{l}$ ), $\mathrm{CD}^{+}$cells were $83 / \mu \mathrm{l}$ (range: $190-1140 / \mu \mathrm{L})$, and $\mathrm{CD}^{+} / \mathrm{CD}^{+}$ratio was 6.82 (range: $0.8-2.0$ ). Complete work-up (including chest radiographs and abdominal and lymph node ultrasound examinations) disclosed no signs of visceral involvement of his KS. The patient reported never hav-

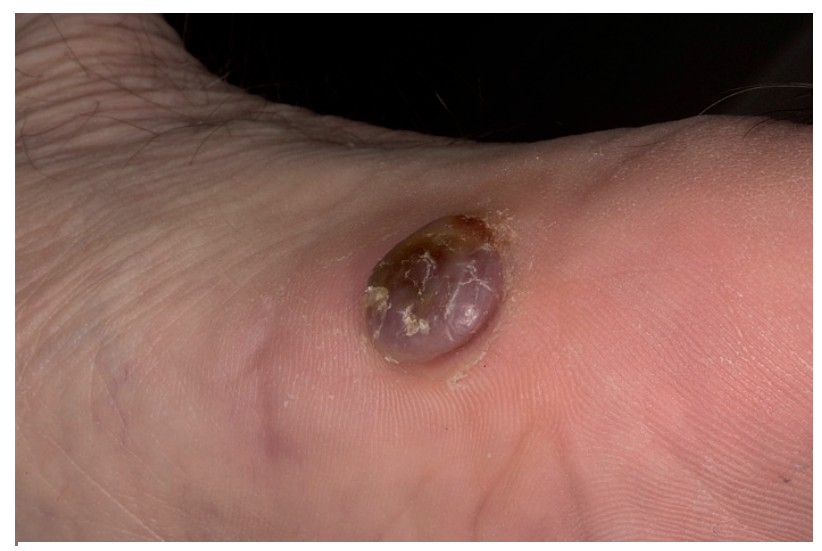

Fig. 1. Clinical presentation of the patient's Kaposi sarcoma. A rapidly growing bluish nodule is located on the patient's left foot.

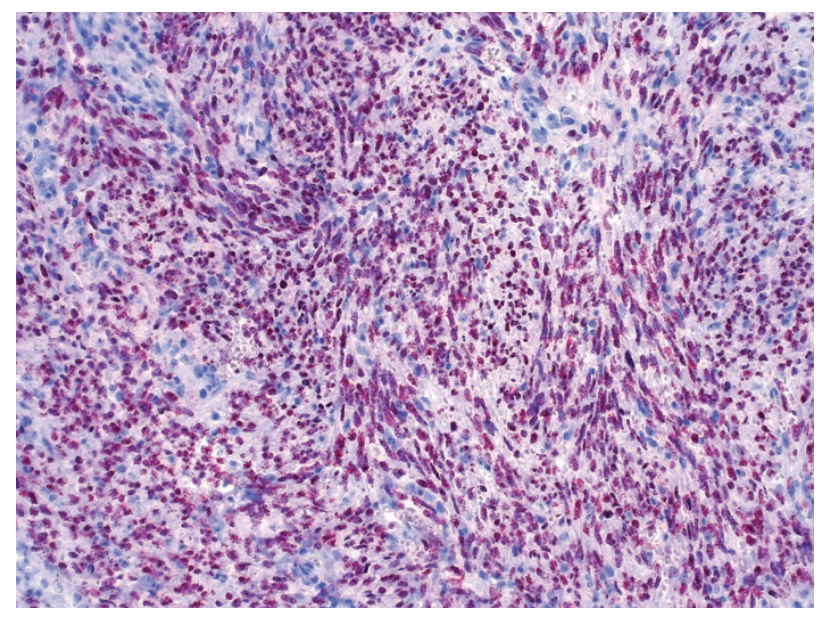

Fig. 2. Immunohistochemical analysis for human herpesvirus 8 (HHV-8) in Kaposi sarcoma. Strong immunoreactivity of HHV-8 is present in the patient's skin lesion (hematoxylin, original magnification x 200). 
ing had receptive and/or insertive anal intercourse, but reported to have frequently engaged in oral sex.

\section{DISCUSSION}

Kaposi sarcoma in HIV-negative MSM has only been reported sporadically, and most of these anecdotal cases were published about two decades ago [1, 2]. However, a French study from 2008 reported on the clinical features and outcome of a total of $28 \mathrm{HIV}$ negative MSM with KS [3]. It was shown that KS in these patients clinically resembles classic KS but occurs at a younger age, is limited to the skin, and is associated with a good prognosis. KS of HIV-negative MSM in this study accounted for approximately 9\% out of a cohort of $300 \mathrm{HIV}$-negative individuals with $\mathrm{KS}$, indicating that $\mathrm{KS}$ in these patients is not absolutely rare.

In Northern Europe HHV 8 seroprevalence is low in the general population $(<5 \%)$, in MSM a seroprevalence of up to $25 \%$ has been described. Epidemiologic studies have shown that oral exposure to infectious saliva is the major risk factor for the acquisition of HHV-8 among MSM [4]. This corresponds to our patient's sexual history, as he reported exclusively oral intercourse.

Interestingly, KS has also been observed in MSM with long-time HIV-infection on successful antiretroviral therapy [5]. All nine patients in this report had high $\mathrm{CD}^{+}{ }^{+}$cells and HIV viral loads below the detection limit, and only mild cases of KS restricted to the skin were seen in these people.

Therapeutic options do not differ from classic $\mathrm{Ka}$ posi's sarcoma. Isolated lesions can be treated with local excision, cryosurgery or laser. Further options are topical alitretinoin gel or radiation (30Gy). Systemic treatment with interferon or liposomal doxorubicin is reserved for wide spread disease or systemic involve- ment [6]. Physicians treating MSM should have in mind that KS might affect both HIV-positive and negative MSM, regardless of their cellular immune status.

Founding sources: None.

Conflict of interest: None declared.

\section{REFERENCES}

1 Friedman-Kien AE, Saltzman BR, Cao YZ, et al. Kaposi's sarcoma in HIV-negative homosexual men. Lancet. 1990;335(8682):168-169.

2 García-Muret MP, Pujol RM, Puig L, Moreno A, de Moragas JM. Disseminated Kaposi's sarcoma not associated with HIV infection in a bisexual man. J Am Acad Dermatol. 1990;23:1035-1038.

3 Lanternier F, Lebbé C, Schartz N, et al. Kaposi's sarcoma in HIV-negative men having sex with men. AIDS. 2008; 22(10):1163-1168.

4 Pauk J, Huang ML, Brodie SJ, et al. Mucosal shedding of human herpesvirus 8 in men. N Engl J Med. 2000; 343 (19):1369-1377.

5 Maurer T, Ponte M, Leslie K, et al. Kaposi's sarcoma in HIV-negative men having sex with men. N Engl J Med. 2007;357(13):1352-1353.

6 Potthoff A, Brockmeyer NH. HIV-associated Kaposi's sarcoma: pathogenesis and treatment. JDDG. 2009; 5 (12):1091-1094.

Received: November 9, 2009/ Accepted: January 27, 2010

Address for correspondence:

Anja Potthoff, MD

Department of Dermatology and Allergology

Ruhr-University Bochum

Gudrunstrasse 56

44791 Bochum

Germany

Tel.: +49-234-509-3473

Fax: +49-234-509-2339

E-mail: a.potthoff@klinikum-bochum.de 